\title{
Proceedings of National Heart, Lung, and Blood Institute Pediatric Cardiology Workshop: Pulmonary Hypertension
}

\author{
Edited by WILLIAM F. FRIEDMAN \\ Los Angeles, California
}

Pulmonary hypertension is a common accompaniment of many congenital cardiac lesions, and the status of the pulmonary vascular bed often is the principal determinant of the clinical manifestations, course, and feasibility of surgical treatment. The causes, appearance, and rate of progression of pulmonary vascular obstructive disease are among the most vexing problems faced by clinicians. Accordingly, the National Heart, Lung, and Blood Institute convened a Pediatric Cardiology Workshop concerned with Pulmonary Hypertension, chaired by Dr. Alfred Fishman, to examine the current status of information regarding the fundamental biology of pulmonary blood vessels and the interaction of these blood vessels with abnormal hemodynamic circumstances.

Chairman: Alfred P. Fishman, M.D., Philadelphia, PA.

Cochairmen: William F. Friedman, M.D., Los Angeles, CA., Abraham M. Rudolph, M.D., San Francisco, CA. Norman Talner, M.D., New Haven, CT.

\section{PROGRAM}

Introduction

Claude Lenfant

Session I. Regulation of Pulmonary

Vasculature

Cochairman: Abraham Rudolph

Embryological Development of Pulmonary Circulation

Oxygen Transfer in The Lungs

Organization of Endothelial Cell

Surface of Alveolar Capillaries

Interaction of the Pulmonary Endothelium with Bioactive Substances

Prostaglandins and Leukotrienes

Control of the Perinatal Pulmonary Circulation

Respiratory Gases- $\mathrm{O}_{2}, \mathrm{CO}_{2}, \mathrm{pH}$

Peter Burri

C. Richard Taylor

E. E. Schneeberger

Una Ryan

Kenneth Brigham

Michael Heymann

Ivan McMurtry
Session II. Pathology and Pathophysiology

Cochairman: William Friedman

Persistent Fetal Circulation

Pathophysiology of Congenital Heart Disease

Pulmonary Vascular Lesions in Congenital Heart Diseases

Pulmonary Vascular Lesions in Acquired Heart Disease

Primary Pulmonary Hypertension

Bronchopulmonary Dysplasia

Session III. Interactions of Blood Constituents with Vessel Wall

Cochairman: Norman Talner

Platelet Derived Growth Factors

Growth Factors: Sources and Actions

Factors Affecting Lung Metabolic Function

Endothelium and Neutrophils

Role of Endothelium in Coagulation

Session IV. Clinical Applications-Diagnosis and Therapy

Cochairman: William F. Friedman

Detection of The Location of Changes in Pulmonary Vascular Tone

Diagnosis: Cardiac Catheterization, Quantitative Wedge Angiography and Lung Biopsy

Echo Doppler Evaluations

Treatment of Primary Pulmonary Hypertension

Treatment of Secondary Pulmonary Hypertension

Pulmonary Hypertension at Altitude

Epilogue

Lynne Reid

Julien Hoffman

C. Wagenvoort

J. Michael Kay

Robyn Barst

William Berman

Thomas Deuel

Patricia D'Amore

Norman Gillis

Peter Henson

Douglas Cines

John Linehan

Marlene Rabinovitch

David Sahn

John Reeves

Lewis Rubin

Robert Wolfe

Alfred Fishman

Address correspondence and reprint requests to William F. Friedman, M.D. Department of Pediatrics, MDCC 22-412, UCLA School of Medicine, Los Angeles, CA 90024.

'The workshop was held at Bethesda, MD from September 22-24, 1985.

\section{MEETING SUMMARY}

The meeting's introductory remarks by Dr. Claude Lenfant provided the reminder that for many years following the advent 
of right heart catheterization, studies of pulmonary hypertension focused on the associated hemodynamic changes. In an attempt to unravel pathogenetic mechanisms of pulmonary hypertension, the physiologic and pathologic effects of alveolar hypoxia on the pulmonary circulation was of intense interest. These studies helped to clarify the role of various factors which regulate the pulmonary circulation, such as innervation, humoral mediators, and mechanical properties, but many questions remained unanswered regarding pulmonary hypertension. New interest in pulmonary hypertension was stimulated in the 1970's when pulmonary vasodilators became widely available. Vasodilators proved to have only limited usefulness as a treatment for pulmonary hypertension; however, they have provided additional information regarding the regulation of the pulmonary circulation.

Although we have learned much about the pulmonary circulation, we still do not have a clear understanding of the causes, prevention, and treatment of pulmonary hypertension. Therefore, investigators recently have taken a more multidisciplinary approach to include cell biology, immunology, biochemistry, hematology, pharmacology, and bioengineering in the study of pulmonary hypertension.

The first workshop session was chaired by Dr. A. M. Rudolph and entitled "The Regulation of Pulmonary Vasculature." Dr. Peter H. Burri discussed the "Embryologic Development of The Pulmonary Circulation." He described the appearance of a lung primordium at day 26 of human gestation as a ventral diverticulum in the foregut-region of the endodermal tube. By growing into the surrounding mesenchyme and via successive dichotomous branchings, the endodermally derived epithelial tube preforms the branching pattern of the future airway tree. The pulmonary microvasculature arises in the mesenchyme and is, therefore, of mesodermal origin. Early during organogenesis branches of the sixth pair of aortic arches grow into the lung and establish the arterial connections to the heart. The venous side is formed as a single evagination from the left atrium, which divides several times and connects the pulmonary plexus. Later, the single pulmonary vein stem and its tributaries of the first and second orders are included into the left atrium.

During fetal life the pulmonary circulation is short-circuited through the foreamen ovale and the ductus arteriosus bridging the pulmonary artery and the aorta. The ductus is a remnant of the left sixth aortic arch. At birth these openings are closed and full perfusion of the lung is established. Despite the uptake and exchange of gas, the function of the pulmonary microcirculation is far from being mature. It has to be completely restructured postnatally after completion of bulk alveolar formation.

Scanning electron microscopic studies were described of vascular casts of the rat lung in which analysis of the morphologic aspects of the capillary restructuring showed dramatic changes after birth. At birth and even after alveolar formation all parenchymal septa contain two capillary networks: one on either side of a central layer of connective tissues. In adult lungs most septa possess a single network with sometimes a double capillary strand at the tip and at the base of the interalveolar walls. From the analysis of the scanning electron micrographs and from earlier quantitative data, Burri derived a new model explaining not only the mode of capillary restructuring, but also how the microvascular network expands during growth. The model showed transition from the double layer capillary system to a single layer system in the alveolar septum; capillarization occurred at the cost of connective tissue. An increase in capillary surface area of almost 20 times occurs in the first 4 months of life, with new capillary meshes arising from enlarging holes at the sites of capillary fusion.

Dr. C. Richard Taylor addressed the issue of "Oxygen Transfer in The Lung." He focused on the question of whether a match exists between structure and function in the development of the lung, i.e. the supply of oxygen across the gas exchange surface to the blood, to meet oxygen demands, should match the changing structure design of the lungs during development. Dr. Taylor described collaborative studies with Dr. E. Weibel in which quantification occurred by morphometric techniques of structures determining the diffusing capacity of the lung for oxygen, as well as quantification of the physiologic parameters determining maximal rates of flow across the lung. A comparative approach was utilized to give large differences and mass specific flow rates of oxygen across the lung; in initial studies body size was used to give more than 10 -fold differences in maximal rates of oxygen uptake per gram of tissue. No match was found between the morphometric diffusing capacity of the lung and maximal rates of oxygen flow across the lung. For example, mice and cattle had very similar structural diffusing capacities for oxygen, yet oxygen flows across the mouse lung were 10 times faster. More recently, studies were conducted to measure physiologic parameters necessary to enable calculation of instantaneous $\mathrm{PO}_{2}$ and oxygen content of blood as it passed through the lung. Dogs and goats were compared, which have a 2.5 -fold difference in maximum oxygen uptake. The dog utilized all of the diffusing capacity of its lung with its blood equilibrating with alveolar $\mathrm{PO}_{2}$ just as it exits the lung. The goat, on the other hand, needs only one-third of its transit time for equilibration between alveolar and arterial $\mathrm{PO}_{2}$ to occur. It is clear that more detailed studies are required to quantify instantaneous $\mathrm{PO}_{2}$ of blood in its transit through the lung, in a variety of species, to further analyze the question of whether the structural design of the lung is well matched to its functional requirements for gas exchange.

The "Organization of Endothelial Cell Surface of Alveolar Capillaries" was discussed by Dr. E. E. Schneeberger. Her research focuses on understanding the blood-alveolar barrier structurally. Normal capillary permeability is regulated, in part, by the interaction of circulating plasma proteins with the capillary wall. It has been suggested that the molecular filter at the capillary wall is glycocalyx which covers and lines the channels conducting fluid and hydrophilic molecules through the endothelium. By binding the components of the glycocalyx, plasma proteins fill the spaces within it, and by increasing its filtering efficiency, decrease its permeability.

In a fluorocarbon exchange transfused rat model, albumin was shown to be localized exclusively to the luminal glycocalyx of pulmonary vascular endothelial cells, reducing vascular permeability to native anionic ferritin to control levels. Whether other circulating proteins are bound with the same affinity and whether such binding will have the same effect on vascular permeability as native albumin is conjectural.

The endothelial glycocalyx bears a net negative charge; the distribution of anionic sites on the pulmonary vascular endothelium, however, remains a matter of controversy. This may be due, in part, to the masking of anionic sites by circulating proteins. To investigate this further, studies were undertaken in the fluorocarbon exchange transfused rat in which circulating proteins were removed in a controlled, graded manner. Cationized ferritin was injected intravenously and the length of the luminal membrane to which cationic ferritin was bound was determined morphometrically, as well as the proportion of luminal vesicle diaphragms binding cationized ferritin. There was an indirect relationship between the concentration of circulating protein and the amount of positively charged ferritin bound to the glycocalyx. Furthermore, marked differences were not observed in the degree of binding of ferritin to the thin as opposed to the thick side of the pulmonary capillary. Thus the glycocalyx plays an important role regulating endothelial permeability; the absorption of circulating proteins to glycocalyx renders the underlying endothelium less permeable to other macromolecules.

Dr. Una S. Ryan provided a comprehensive discussion of "Interactions of the Pulmonary Endothelium with Bioactive Substances." Of the so-called metabolic or "nonventilatory" functions of the lungs, many in fact can be attributed to pulmonary endothelial cells. Thus the processing of vasoactive 
substances (e.g. biogenic amines, adenine nucleotides, prostaglandins, and peptide hormones) is mediated by specific enzymes, enzyme inhibitors, receptors, or transport molecules situated on the luminal surface of pulmonary endothelial cells. Although the enzymes may occur in plasma or in or on blood elements, the physiologically relevant pulmonary processing frequently can be accounted for by the endothelial-disposed enzyme. Because of the enormous surface area of the pulmonary capillary bed and the brief transit times $(2-3 \mathrm{~s})$ it is clear that these are extremely rapid and efficient reactions. In the case of the conversion of angiotensin I to angiotensin II there is more than ample enzyme to provide for physiologic and some pathologic exigencies without compromise. Yet the disposition of enzyme can provide a basis for the estimation of pulmonary vascular surface area.

Interaction of the pulmonary endothelium and bioactive substances determines the levels of these substances allowed to circulate in systemic blood and influences the functions of target organs and tissues at a distance. Thus the lungs, through the activities of their endothelial cells, play a role in a wide range of hemostatic mechanisms including the control of blood pressure, hemostasis, salt and water balance, $\mathrm{CO}_{2}$, and $\mathrm{pH}$ balance. However, some interactions of reactive substances carried in the blood have local effects within the lungs themselves. Pulmonary endothelial cells are not only metabolically active and ultrastructurally complex but they are also highly responsive to bloodborne effectors. For example, bradykinin, a substance degraded by pulmonary endothelial cells, is also capable of acting upon endothelial cell receptors to elicit an increase in $\mathrm{PGI}_{2}$ synthesis. Other substances (inflammatory mediators and complement components) provide subtle injuries or "stimuli" that may alter the actively antithrombogenic and immunologically unreactive endothelial surface into one fully capable of expressing procoagulant activities and of expressing $\mathrm{Fc}$ and $\mathrm{C} 3 \mathrm{~b}$ receptors. Thus endotoxin can stimulate tissue factor expression and can cause the synthesis of an inhibitor of plasminogen activator. In addition, it causes unmasking of latent Fc receptors on endothelial cells. Interleukin 1 also is capable of causing an increase in expression of endothelial tissue factor activity. Similarly, phagocytosis of particulate material induces an activation of endothelial cells and unmasking of $\mathrm{Fc}$ receptors on the endothelial surface. The endothelial glycocalyx or surface coat becomes disarrayed by the same conditions that accompany changes in function and appears to provide the structural correlate of the changes in endothelial hemostatic and immunologic potential.

Ryan emphasized that pulmonary endothelial cells are actively engaged in the regulation of systemic levels of vasoactive substances, presumably as a result of constant interaction of circulating substances with the endothelial surface. In addition, episodic interaction of pulmonary endothelial cells with bloodborne stimuli, including engulfment of particulate matter, can provide a means whereby endothelial cells actively participate in events leading to vascular occlusion and damage and can provide conditions for procoagulant and complement linked activities in the inflammatory response.

The "Regulation of Pulmonary Vasculature by Prostaglandins and Leukotrienes" was discussed by Dr. Kenneth L. Brigham. Metabolites of arachidonic acid include the most potent known constrictor and dilator substances in the lung circulation. At least the synthetic thromboxane $A_{2}$ mimic (and probably thromboxane $A_{2}$ itself) is an extremely potent pulmonary vasoconstrictor; doses of the agent which triple pulmonary vascular resistance have little effect on the systemic vasculature. Prostacyclin is a very potent pulmonary vasodilator. Biologically active metabolites of arachidonic acid are produced in the lungs and have attracted attention as potential mediators of alterations in vascular tone and vascular reactivity.

Infusion of arachidonic acid intravenously into experimental animals causes vasoconstriction which is largely prevented by cyclooxygenase inhibitors. This suggests that under baseline cir- cumstances the cyclooxygenase pathway is the predominant route of arachidonate metabolism. The response to arachidonic acid appears to be purely vasoconstriction; i.e. there is no evidence of any effects on pulmonary vascular permeability.

Several cyclooxygenase products of arachidonate are pulmonary vasoconstrictors including $\mathrm{PGE}_{2}, \mathrm{PGF}_{2}, \mathrm{PGB}_{2}$, and $\mathrm{PGE}_{2}$. Some lipoxygenase products, especially leukotriene $\mathrm{D}_{4}$ and to a lesser extent leukotriene $\mathrm{C}_{4}$, also are pulmonary vasoconstrictors, although in the case of leukotriene $\mathrm{D}_{4}$, this effect is at least partially inhibited by cyclooxygenase inhibitors, indicating a complex effect. The pulmonary vasoconstriction resulting from endotoxemia in sheep appears to be mediated partially by a cyclooxygenase products of arachidonic acid since the response is moderated by cyclooxygenase inhibitors. The response also is accompanied by increased concentrations of a thromboxane metabolite in lung lymph and is moderated by more specific inhibitors of thromboxane synthesis, both tending to implicate thromboxane $\mathrm{A}_{2}$ as the mediator. However, recent data show that leukotrienes (at least $\mathrm{LTB}_{4}$ ) are released in the lungs during the early part of the endotoxin response, so that lipoxygenase products also could be involved in the pulmonary vasoconstrictor response to endotoxemia.

Much interest has focussed on the possible role of arachidonic acid metabolites in mediating hypoxic pulmonary vasoconstriction (constrictor products) and loss of this response (dilator products). Recent studies seem to implicate lipoxygenase products as mediators in the constrictor response in isolated perfused lungs. Hypoxic vasoconstriction is lost following diffuse lung inflammation in several animal models, and in some of these models the response is restored by treatment with cyclooxygenase inhibitors. This has led to the hypothesis that vasodilator products of arachidonate are responsible for preventing the hypoxic response, but not all experimental evidence supports this hypothesis.

The "Control of the Perinatal Pulmonary Circulation" was analyzed by Dr. Michael A. Heymann. In the fetus pulmonary vascular resistance is very high and pulmonary blood flow is only about $30-40 \mathrm{ml} / \mathrm{kg}$ body weight $/ \mathrm{min}$ or about $8 \%$ of combined ventricular output. After birth and the initiation of pulmonary ventilation pulmonary vascular resistance falls rapidly and pulmonary blood flow (now equal to cardiac output) increases 810 -fold to about $350-400 \mathrm{ml} / \mathrm{kg} / \mathrm{min}$. There is a further slow progressive fall in pulmonary vascular resistance over the next 6-8 wk. Maintenance of low fetal pulmonary flow is due, at least in part, to the normally present low $\mathrm{O}_{2}$ environment $\left(\mathrm{PaO}_{2} 20\right.$ 30 torr) maintaining pulmonary vasoconstriction. Recent evidence suggests a role for leukotrienes, the 5-lipoxygenase products of arachidonic acid metabolism, in maintaining fetal pulmonary vasoconstriction as well, particularly leukotriene $\mathrm{C}_{4}$. The role of other vasoactive substances is less clearly established. The dramatic pulmonary vasodilation that occurs after birth is affected by several phenomena. The effect of increasing $\mathrm{O}_{2}$ environment is clearly established, but whether this is a primary response is unclear.

The local production and release of several vasoactive substances also is established. Bradykinin is released and is a potent but nonspecific vasodilator. The cyclooxygenase products of arachidonic acid metabolism (the prostaglandins) likely play a role as well. Inhibition of PG synthesis with indomethacin attenuates the postnatal pulmonary vasodilatation. The stable metabolite of $\mathrm{PGI}_{2}$, a fairly potent but also nonspecific vasodilator, is released from the lungs in the immediate postnatal period, probably due to the mechanical effects of lung expansion. Mast cell concentration in the lung increases toward term in the fetus and falls after birth suggesting mast cell degranulation after birth. Mast cells release histamine and $\mathrm{PGD}_{2}$, both pulmonary vasodilators in the early postnatal period only. Disturbances in the relationship between vasoconstricting and vasodilating substances after birth likely play a major role, together with induced morphologic changes in the resistance vessels, in failure of the 
establishment of normal postnatal pulmonary blood flow or the syndrome of persistent pulmonary hypertension of the newborn. Medical management of these infants has taken into account many of the above physiologic observations but, unfortunately, all substances used so far are nonspecific vasodilators and have not been effective.

Dr. Ivan F. McMurtry discussed the "Regulation of The Pulmonary Circulation by The Respiratory Gases, $\mathrm{O}_{2}$ and $\mathrm{CO}_{2}$, and Blood pH," which has been the subject of numerous studies since 1946 when Euler and Liljestrand described hypoxic and hypercapnic pulmonary vasoconstriction in anesthetized cats. There is interest in the mechanism of hypoxic pulmonary vasoconstriction (HPV), and its potentiation by hypercapnic acidosis, because of the role of HPV in the shunting of blood flow from the unventilated fetal lung and the reversal of this process in newborns, the local matching of pulmonary capillary blood flow to alveolar ventilation in the adult, and the development of pulmonary hypertension and right heart failure in patients with chronic ventilatory insufficiency. Study of HPV is also relevant to vascular smooth muscle and endothelial cell physiology and to the biochemistry of $\mathrm{O}_{2}$ sensing.

Although the magnitude of HPV is influenced by systemic hemodynamic, neural, and humoral signals, the basic mechanism comprising $\mathrm{O}_{2}$ sensor, transduction process, and effector is evident in isolated lungs perfused with physiologic salt solution, i.e. the basic mechanism is intrapulmonary. Smooth muscle of the distal pulmonary arteries is probably the primary effector, but the cellular site and molecular nature of the $\mathrm{O}_{2}$ sensor and the biochemical chain of events by which the sensing of hypoxia is converted to constriction of vascular smooth muscle are uncertain. A current belief is that HPV is caused either by elaboration of a chemical mediator from some lung cell or by a direct excitatory effect of hypoxia on the vascular smooth muscle.

Numerous studies have provided evidence that neither catecholamines, histamine, serotonin, prostaglandins, angiotensin II, vasopressin, bradykinin, acetylcholine, substance P, VIP, lactic acid, nor ATP is the mediator of HPV. A current candidate is leukotrene (LT) $\mathrm{C}_{4}$, a vasoconstrictive product of arachidonic acid metabolism by the lipoxygenase pathway. Evidence is that $\mathrm{LTC}_{4}$ is found in the airway lavage of hypoxic rat lungs and that $\mathrm{HPV}$ is inhibited, at least in some studies, by putative blockers of $\mathrm{LTC}_{4}$ synthesis (DEC, U-60257), of release (cromolyn sodium), and of action (FPL 55712 and 57231). However, it should be noted that this evidence is similar to that used $10 \mathrm{yr}$ ago to propose histamine as the mediator of HPV. Further work is necessary to substantiate the role of $\mathrm{LTC}_{4}$ in the mechanism of HPV.

A possible sequence for a direct effect is that hypoxia acts on the vascular smooth muscle to decrease mitochondrial oxidative phosphorylation and alter the energy state $($ e.g. $[\mathrm{PCr}] /[\mathrm{Cr}]$ or $[\mathrm{ATP}] /[\mathrm{ADP}][\mathrm{Pi}])$, which leads in some way to membrane depolarization, $\mathrm{Ca}^{++}$influx, and contraction. Evidence for involvement of some pertubation of energy metabolism is provided by observations in isolated lungs that chemical inhibitors of oxidative phosphorylation such as azide, antimycin, cyanide, DNP, and rotenone cause $\mathrm{Ca}^{++}$-dependent vasoconstriction during normoxia, and that inhibition of glucose metabolism by iodoacetate, 2-deoxyglucose, or glucose-free perfusion increases hypoxic pressor sensitivity. Inhibition of hypoxic vasoconstriction in isolated lungs by the $\mathrm{Ca}^{++}$channel blockers verapamil and nifedipine, and potentiation by the $\mathrm{Ca}^{++}$channel facilitator BAY K 8644 support the idea that activation of voltage-dependent $\mathrm{Ca}^{++}$channels is a component of the mechanism of HPV. Further evidence is provided by recent studies showing that the $\mathrm{Ca}^{++}$-dependent hypoxic contraction of small pulmonary arteries isolated from the cat is associated with smooth muscle membrane depolarization and generation of action potentials. The establishment of this latter preparation as a valid in vitro model of HPV will provide the means to more directly test the above hypotheses and the possible involvement of endothelial cells, cytochrome p-
$450 \mathrm{O}_{2}$-derived free radicals, and lipid peroxidation in the mechanism of HPV.

Session II was entitled "Pathology and Pathophysiology" and was chaired by Dr. William F. Friedman. To understand the varieties of "Persistent Fetal Circulation (PFC)" seen clinically, Dr. Lynne Reid discussed the necessity to consider first the structure and growth of the pulmonary circulation before birth and its perinatal adaptation. The well-recognized functional adaptation of a drop in pressure and resistance is based on dilatation and also on the structural change of increased compliance of the precapillary resistance units. The structural features special to pulmonary microcirculation were described in terms of the preand postcapillary unit at term and during subsequent perinatal adaptation.

Dr. Reid proposed that three different types of PFC can be identified: (a) failure of perinatal adaptation, as in certain types of congenital heart disease and congenital lung anomalies; (b) abnormal development of the pulmonary circulation with precocious muscularization of the precapillary unit, as seen in the idiopathic variety of PFC and also in fatal cases of meconium aspiration; and (c) hypoplasia or immaturity of the pulmonary circulation which is demonstrated by correction of the lung after congenital diaphragmatic hernia. The pulmonary circulation at first accepts a full cardiac output and maintains normal blood gases. In some of these patients the circulation is labile, i.e. constriction occurs that causes right-to-left shunting, and although at first it is labile, it then becomes fixed and fails to respond to conventional dilators. In these instances, sedation has proved more effective than dilatation. Presumably, hypoplasia can be so extreme that the pulmonary vascular bed can neither accept the full cardiac output nor maintain blood gases. Cases of congenital diaphragmatic hernia illustrate all three mechanisms.

Dr. Julien Hoffman provided a discussion of "Pulmonary Vascular Disease," one of the major complications of congenital heart disease. Table 1 gives estimates based on current knowledge about the incidence of pulmonary vascular disease in untreated congenital heart disease. The incidence is a maximal incidence because some of these patients would die for various reasons before pulmonary vascular disease had time to occur. Nevertheless, Table 1 makes the point that when all other causes of death have been eliminated, about one-third of these patients could eventually die from pulmonary vascular disease.

The histopathology of pulmonary vascular disease has been described in two phases-early descriptions in the 1950's of what is termed the Heath-Edwards classification and then extensions of this by Reid and her colleagues in the 1970's. Unfortunately, our understanding of the physiology of pulmonary vascular disease lags behind our knowledge of its morphology.

Stage 1 of pulmonary vascular disease is an increase in the muscle in the media of the small pulmonary arteries and arterioles. Not only does this medial thickening occur, but medial smooth muscle extends more distally in the vascular bed. Normally at birth in a term infant the small pulmonary arterial vessels are muscularized only as far as the beginning of the respiratory bronchiole, and it is only by the late teens that smooth muscle spreads down to the intraalveolar arteries. In pulmonary vascular disease this distal extension is speeded up and may be complete by 1 or 2 yr. The likely cause of the muscular hypertrophy is the increase in pressure or volume work of the arterial wall, so that increased muscularity is found with any sustained increase in pulmonary blood flow-as in large atrial septal defects-but more particularly when there is pulmonary hypertension, whether or not pulmonary blood flow is also increased. Whether smooth muscle cells divide and migrate distally, form in situ from precursor cells, or both is not established. When the stimulus to muscle growth is removed, the media does become thinner. Whether regression of abnormal distal muscularization occurs or whether the distal muscle extension remains until it comes to be normal for age with the passage of years is unknown.

Little is known about the reactivity of these abnormally mus- 
Table 1. Pulmonary vascular disease*

\begin{tabular}{|c|c|c|c|}
\hline Lesion & $\%$ & Total no. & No. at risk \\
\hline Ventricular septal defect & 30 & 9000 & 3000 \\
\hline Patent ductus arteriosus & 9 & 2700 & 900 \\
\hline Atrial septal defect & 7 & 2100 & 700 \\
\hline Atrioventricular septal defect & 3 & 900 & 800 \\
\hline Aortic stenosis & 5 & 1500 & 0 \\
\hline Pulmonic stenosis & 7 & 2100 & 0 \\
\hline Coarctation & 6 & 1800 & 0 \\
\hline Tetralogy & 5 & 1500 & 200 \\
\hline \multirow[t]{2}{*}{ Transposition of the great arteries } & 5 & 1500 & 500 \\
\hline & 1 & 300 & 300 \\
\hline Hypoplastic right heart & 2 & 600 & 50 \\
\hline Hypoplastic left heart & 1 & 300 & 0 \\
\hline Double outlet right & 0.2 & 60 & 60 \\
\hline Total anomalous pulmonary venous connection & 1 & 300 & 300 \\
\hline Univentricular heart & 0.3 & 90 & 100 \\
\hline Miscellaneous & 17.5 & 5250 & 2625 \\
\hline Total & 100.0 & 30,000 & $9535(32 \%)$ \\
\hline
\end{tabular}

* Assumptions: $3,000,000$ live births per year; $1 \%$ incidence of congenital heart disease.

cularized vessels. Medial muscular hypertrophy is usually accompanied by an increased pulmonary vascular resistance that can be reduced by vasodilators. However, the increased muscularization in atrial septal defects is associated with a very low pulmonary vascular resistance. We do not know how important distal muscle extension is in increasing pulmonary vascular resistance, or if the new smooth muscle cells have their full complement of receptors and are as reactive as are the mature muscle cells.

With progression of the pulmonary vascular disease intimal changes occur. First, there is a piling up on the endothelial surface of amorphous material that may include platelet debris and this is followed by intimal hyperplasia. The origin of the fibroblast-like cells that appear in the intima still is disputedthey may come from medial smooth muscle cells that migrate and change, or they may arise from mesenchymal cells present in the intima. This stage II of pulmonary vascular disease then progresses until the intima becomes not only thick but also hyalinized and with relatively few nuclei; this is stage III. Stage IV is an acute arteritis and the peculiar angiomatous, plexiform, and dilatation lesions of stages IV and V may be sequelae of the acute arteritis. Finally, and most importantly, progression of pulmonary vascular disease by these Heath-Edwards stages is accompanied by failure of growth of adequate numbers of small pulmonary arteries so that the arterial:alveolar ratio falls progressively as pulmonary vascular disease advances. As a result not only are the small pulmonary arteries thickened with small lumens, but there are fewer of them than expected.

Dr. Hoffman emphasized that our understanding of the mechanisms involved in these changes is imperfect, leading to the following questions and comments: 1. Are the intimal lesions always consequences of endothelial damage? An increased shear stress has been shown to damage endothelium acutely. Is this always the mechanism or are other factors involved? 2. Endothelial damage followed by platelet aggregation could initiate the changes from stage II onward. Is the intimal change in pulmonary vascular disease similar to the early atheromatous changes in systemic arteries? Are platelet-derived growth factors-or other growth factors-responsible for the intimal hyperplasia? If so, can hyperplasia be prevented or retarded by preventing platelet aggregation or blocking receptors for growth factors? 3. Is platelet aggregation a sustaining rather than an initiating mechanism of pulmonary vascular disease? Some patients have evidence of platelet consumption including increased serum levels of platelet factor IV, platelet-derived thromboglobulin, and fibrin split products. Would agents that prevent platelet aggregation retard the advance of pulmonary vascular disease? 4 . Is some of the vasoconstriction observed when there is pulmonary vascular disease due to reduced production of endothelial derived relaxing factor? 5. Acute arteritis has occurred experimentally after the onset of acute pulmonary hypertension. Are there other causes? How can it be prevented? 6 . Why do new pulmonary arteries not form at the normal rate? Can their growth be stimulated? Up to what age will they regrow if the basic congenital heart lesion is corrected? How can we measure the number of small pulmonary arteries without biopsy?

The conclusion of Dr. Hoffman's presentation stressed that our emphasis must shift from histopathology to cell biology-of endothelial, mesenchymal, and smooth muscle cells and platelets, as well as interactions among these cells. Only with this knowledge can prospects of prevention and treatment of pulmonary vascular disease be accomplished.

Dr. C. A. Wagenvoort next presented his views of the "Vascular Lesions in Congenital Heart Disease." Generally the pathologic alterations in pulmonary blood vessels may predict the nature of the hemodynamic changes resulting from congenital heart disease. The most familiar pattern of pulmonary vascular lesions in congenital heart disease, more particularly in the presence of a left-to-right shunt, is plexogenic arteriopathy. It consists of a sequence of lesions beginning with medial hypertrophy, followed by cellular proliferation, and concentric laminar intimal fibrosis. Eventually the most advanced alterations (dilatation lesions, fibrinoid necrosis, and plexiform lesions) develop. Although this sequence is likely to be adhered to in principle, there is great variation with regard to the severity of individual changes. Plexiform lesions, for instance, may develop in the presence of very mild intimal fibrosis but sometimes also when there is extensive obstruction by intimal fibrosis.

The course of progression of the lesions may vary from some months to many years. Death from right cardiac failure may occur when there are no lesions more severe than intimal fibrosis or, in infants, even than medial hypertrophy. Rapid progression is particularly observed in complicated cardiac defects such as transposition of the great arteries with a ventricular septal defect, but an individual reactivity of lung vessels may well play an important part. Reversibility of plexogenic arteriopathy applies to medial hypertrophy and to the intimal lesions as long as intimal fibrosis has not become severe.

Several other forms of pulmonary vascular disease may occur in congenital heart disease. The vascular alterations of pulmonary venous hypertension are to be found as the predominant changes in cardiac anomalies such as congenital mitral stenosis 
or atresia, aortic atresia, cor triatriatum, or total anomalous pulmonary venous connection. The pulmonary arteries show severe medial hypertrophy and eccentric intimal fibrosis, while medial thickening and arterialization occur in the pulmonary veins. These lesions are likely to be essentially reversible. Similar changes also may complicate the patterns of plexogenic arteriopathy. This happens in mitral valve incompetence in association with an atrioventricular septal defect and also may result from left ventricular failure in the later stages of congenital heart disease with a shunt.

Postthrombotic changes, more likely to be primary than embolic, are not uncommon in congenital heart disease but particularly occur in complete transposition of the great arteries. The lesions consist of eccentric intimal fibrosis due to organization of thrombi and of intravascular septa due to their recanalization.

If congenital heart disease results in a diminished pulmonary arterial pulse pressure, the media of the arteries tends to become very thin. This medial atrophy is common in pulmonic stenosis and tetralogy of Fallot. Particularly in the latter condition it is associated often with postthrombotic lesions, e.g. eccentric intimal fibrosis as well as very delicate intravascular septa.

The "Pulmonary Vascular Lesions in Acquired Heart Disease" were the subject of a presentation by Dr. J. Michael Kay. Apart from very rare conditions such as a ruptured interventricular septum following myocardial infarction, acquired heart diseases leading to pulmonary vascular disease have one basic physiological abnormality-pulmonary venous hypertension. Since there are no valves in the pulmonary veins, an increase in the left atrial pressure may be passively transmitted back through the pulmonary capillaries to elevate the pulmonary artery pressure. An increase in the left atrial pressure of more than $20 \mathrm{~mm} \mathrm{Hg}$ seems to cause reflex vasoconstriction in the pulmonary arteries and a disproportionate rise in the pulmonary artery pressure. The acquired heart lesions which may cause chronic left atrial hypertension include mitral stenosis, mitral incompetence, left atrial myxoma, and any cause of chronic left ventricular failure, including ischemic heart disease, systemic hypertension, aortic stenosis, aortic incompetence, and the various forms of myocarditis and cardiomyopathy.

Chronic pulmonary venous hypertension produces a characteristic combination of changes in the pulmonary arteries and arterioles, pulmonary capillaries, pulmonary veins, and lung parenchyma. The pulmonary arterioles become muscularized with the development of a distinct tunica media bounded between internal and external elastic laminae. Such vessels which measure less than $100 \mu$ in external diameter are normally devoid of histologically discernible smooth muscle and consist of a single elastic lamina lined by endothelial cells. The muscular pulmonary arteries show medial thickening and intimal fibrosis. In contrast to other forms of pulmonary hypertension, in pulmonary venous hypertension there is a regional distribution of the vascular lesions, probably related to the higher hydrostatic pressure in the lower parts of the lungs. In mitral stenosis the medial thickening of pulmonary arteries is more pronounced at the bases of the lungs than in the apices. On the other hand, intimal fibrosis often is more conspicuous in the upper parts of the lungs. In adults with mitral stenosis the number of smooth muscle cell nuclei per unit medial surface area of pulmonary arteries is reduced, whereas it is increased in children.

Ultrastructural studies have shown an increase in the amount of collagen in the tunica media of pulmonary arteries of patients with mitral stenosis. In contrast to congenital heart disease, adult patients with mitral stenosis do not show a correlation between the pulmonary artery pressure, on the one hand, and the medial thickness and severity of intimal fibrosis of the pulmonary arteries on the other hand. Intimal fibrosis usually is prominent in pulmonary arteries. It may be either concentric or eccentric, but it does not show the laminar and onion-skin pattern which is characteristic of the pulmonary hypertension complicating ventricular septal defect. Ultrastructural studies have shown that the principal cell in the subendothelial intimal layer is the smooth muscle cell. Obliteration of the pulmonary arteries by intimal fribrosis is uncommon. Fibrinoid necrosis and arteries are rare. Plexiform lesions and dilation lesions have not been observed in chronic pulmonary venous hypertension.

Electron microscopy of the alveolar-capillary wall in patients with mitral stenosis has shown edematous swelling of the capillary endothelial cells and thickening of their basal laminae, which sometimes enclose fragments of extravasated erythrocytes. Interstitial edema is confined to the thicker collagen-containing portions of the alveolar wall and does not involve the thinnest portions of the blood-air barrier which are devoid of collagen and situated over the convexities of the alveolar capillaries. A proliferation of dense connective tissue surrounds some of the alveolar capillaries and appears to displace them inward from their normal superficial position in the alveolar wall.

The walls of normal pulmonary veins consist of a haphazard arrangement of elastic fibrils, smooth muscle cells, and collagen. Normally, there is no clear demarcation between the media and adventitia. In chronic pulmonary venous hypertension the pulmonary veins show medial hypertrophy. They also show a change, referred to as "arterialization," in which a distinct tunica media and arterialization of pulmonary veins are much more prominent at the base of the lung than at the apex. This is in contrast to intimal fibrosis, which is more marked at the apex than at the base, a situation resembling that in the pulmonary arteries.

In chronic pulmonary venous hypertension there is dilatation of the lymphatic channels which run in the perivascular, peribronchial, and interlobular fibrous tissue. There also are characteristic changes in the lung parenchyma including interstitial fribrosis, hemosiderosis, and bone metaplasia. In the areas of interstitial fibrosis there is a proliferation of granular pneumocytes leading to the appearance of cuboidal cell metaplasia of alveolar walls.

In most patients with mitral stenosis the elevated pulmonary vascular resistance falls rapidly to almost normal after mitral valve surgery. It is rare for a pathologist to be able to compare the pulmonary vascular lesions at lung biopsy during mitral valve surgery with the situation in the same patient many years later. There are occasional reports of the regression of pulmonary vascular changes following successful correction of mitral stenosis. Recently, a series of 16 patients who died following mitral valve replacement was described. It was concluded that some patients with mitral stenosis who die after surgery with persistently elevated pulmonary artery pressures have sufficiently severe pulmonary vascular disease to account for their persistent pulmonary hypertension and death.

Dr. Robyn J. Barst next discussed her studies of "Primary Pulmonary Hypertension." It is well known that adults with primary pulmonary hypertension often have severe plexiform lesions and fixed pulmonary vascular changes. On the other hand, pathologic studies show greater medial hypertrophy and less intimal fibrosis and plexiform lesions in younger patients with primary pulmonary hypertension. These postmortem studies suggest that pulmonary vasoconstriction leading to medial hypertrophy occurs early in the course of the disease and precedes the development of plexiform lesions and fixed pulmonary vascular changes. Based on the hypothesis that hyperreactivity is a part of the early pathogenesis of primary pulmonary hypertension, Barst administered intravenous prostacyclin to nine children and young adults with primary pulmonary hypertension $(9$ months to $23 \mathrm{yr}$ ) to assess pulmonary vasoreactivity, i.e. the extent to which prostacyclin infusion acutely reverses pulmonary hypertension. In addition, whether vasoreactivity predicted the response to other vasodilators was investigated. Because recruitment or passive distension of pulmonary vessels can decrease pulmonary vascular resistance without necessarily indicating a decrease in pulmonary vascular tone and because spontaneous hemodynamic variability is known to occur in some patients 
with primary pulmonary hypertension, strict criteria were used to indicate drug-induced pulmonary vasodilatation. In this group of patients with primary pulmonary hypertension patients who had a reactive pulmonary vascular bed, defined by their response to prostacyclin, also dilated their pulmonary circulation in response to nifedipine. In addition, the youngest subjects demonstrated the largest component of reversible pulmonary hypertension, supporting the concept that vasoconstriction may be an early event in the pathogenesis of primary pulmonary hypertension (Fig. 1).

Having identified pulmonary vasoreactivity, several mechanisms that may be contributing to the maintenance or exacerbation of pulmonary vasoconstriction and pulmonary hypertension were examined, including the possible contributions of the sympathetic nervous system and the arachidonic acid cascade to the vasoreactivity present in some of the patients. Several patients had increased circulating levels of norepinephrine or thromboxane, both pulmonary vasoconstrictors. Pharmacologic attempts at antagonizing these vasoconstrictors has resulted in prolonged survival and decreased pulmonary hypertension in some of the patients. Barst suggested that pulmonary vasoconstriction and hyperactivity are key components in the pathogenesis of primary pulmonary hypertension, and that multiple factors lead to the increased vasoreactivity. Although it is uncertain at what stages the pulmonary vascular lesions in primary pulmonary hypertension are reversible, her studies suggest that at least in some patients they are. The use of vasoactive drugs such as prostacyclin may help define those patients in whom the pulmonary vascular lesions are reversible.

"Bronchopulmonary Dysplasia" (BPD) was discussed by Dr. William Berman who followed 10 children for an average of 4.4 $\mathrm{yr}$, evaluated initially by cardiac catheterization (mean age 18 months). The children resided at an altitude of $5000 \mathrm{ft}$. Their age at last evaluation averaged $5.8 \mathrm{yr}$. At initial cardiac catheterization, the mean pulmonary artery pressure averaged $40 \mathrm{~mm}$ $\mathrm{Hg}$ and the pulmonary vascular resistance index $8.9 \mathrm{U}$; intrapulmonary shunt fractions were high and pulmonary wedge angiograms were normal. Over the period of follow-up the children grew poorly (mean height 22 nd percentile, mean weight 21 st percentile); four of the 10 remained on home $\mathrm{O}_{2}$ therapy, but none required inotropic or diuretic therapy; four children had marked developmental/motor delays. Nine of 10 patients had abnormalities of respiratory function on spirometric testing. Four patients were recatheterized for clinical indications; two of them had large atrial level left-to-right shunts not found on initial study. Reduction in mean pulmonary artery pressure (55 to 37 $\mathrm{mm} \mathrm{Hg}$ ) and pulmonary vascular resistance $(11.9$ to $7.8 \mathrm{U})$ occurred between the two studies in these four patients (average study interval $4.0 \mathrm{yr}$ ); the still elevated levels of pressure and resistance fell further in response to $40 \% \mathrm{O}_{2}$ administration (to $27 \mathrm{~mm} \mathrm{Hg}$ and $4.9 \mathrm{U}$, respectively). Pulmonary wedge angiograms were abnormal in each restudied patient. Thus these findings suggest, although not uniformly bleak, the long-term outlook for children with severe BPD is diverse and guarded.

Session III, chaired by Dr. Norman Talner, was entitled "Interactions of Blood Constituents with Vessel Wall."

Dr. Thomas F. Deuel next discussed "Platelet-Derived Growth Factor (PDGF)" which is the most potent mitogenic protein in human serum. The normal roles of this protein may relate to its potent mitogenic properties and its activities in directed cell migration and at sites of wounds. PDGF is a heterodimeric protein of about 30,000 molecular weight; one polypeptide chain of PDGF is highly homologous to the predicted amino acid sequence of $\mathrm{p} 28^{\mathrm{v}-\mathrm{sis}}$, the putative transforming protein of simian sarcoma virus (SSV), suggesting a major role of growth factor activity in transformation by SSV. PDGF-like growth promoting activity is found in SSV-transformed cells and is secreted into conditioned media where it appears to interact with PDGF cell surface receptors to stimulate ${ }^{3} \mathrm{H}$-thymidine incorporation into DNA of the cells secreting this protein. Transformation by SSV, in part may, be mediated by the autocrine stimulation of cell growth by the PDGF-like mitogenic properties of the transforming protein of SSV.

Cell growth and division are highly regulated and highly conserved processes that are disrupted in neoplasia, resulting in uncontrolled growth which is characteristic of the cancer cell. Neoplastic or transformed cells generally are represented as having a block in normal cellular differentiation or by undisciplined cell growth. The basis of the uncontrolled cell growth in neoplasia is poorly understood, but the development of detailed knowledge of the metabolic pathways and molecules that regulate normal cell growth should clarify the mechanism of abnormal growth in cancer cells. A group of polypeptide growth promoting factors recently have been isolated and characterized and shown to regulate the proliferation and survival of normal cells. Although the mechanisms of action and in vivo function of these polypeptide growth factors are not established, it is likely that growth factors alone, or working in conjunction with one an-

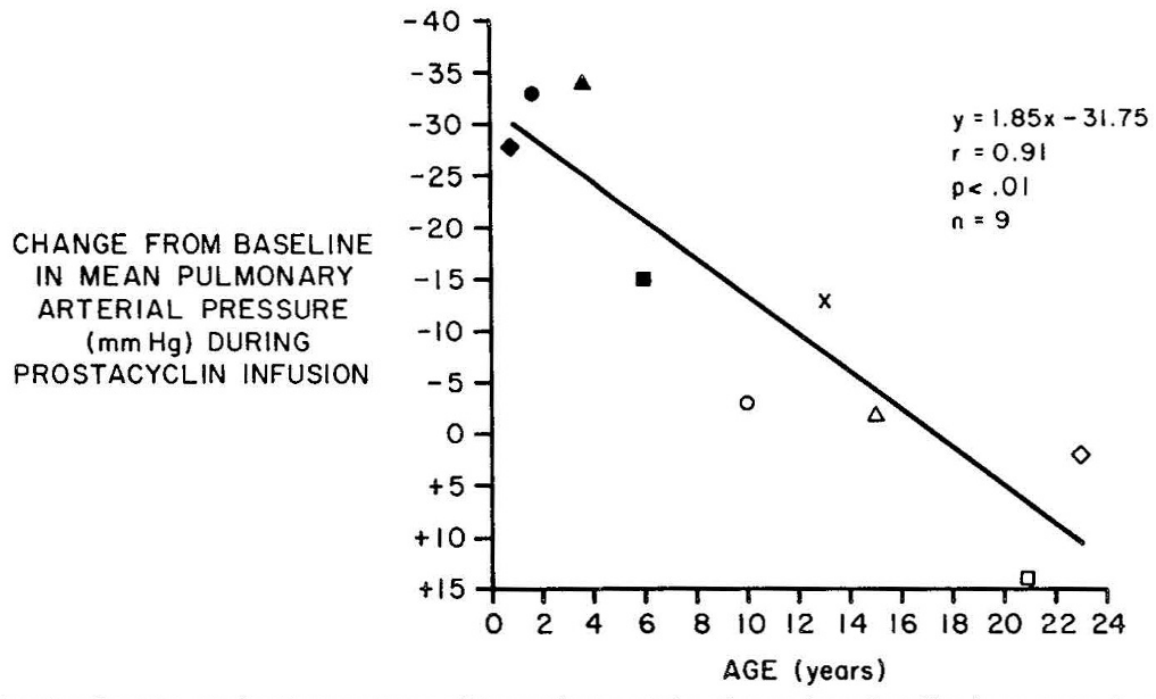

Fig. 1. Dr. Barst's results showing the relation between age of the patient and the change from baseline in mean pulmonary arterial pressure with prostacyclin infusion. The significant inverse correlation indicates that prostacyclin produces a greater fall in pulmonary arterial pressure in younger patients with primary pulmonary hypertension than in older ones. A similar inverse correlation $(R=0.82, p>0.01)$ was observed between the age of the patient and the change from baseline in mean pulmonary arterial pressure with sublingual nifedipine administration. 
other, have major roles in normal cell development, cell differentiation, and tissue repair. The growth factors were identified first by their effects of increased DNA synthesis and cell proliferation. Importantly, growth factor-stimulated cells assume characteristics similar to characteristics of cells transformed by retroviruses. Similarly, transformed cells synthesize and secrete polypeptide growth factors that have the potential to stimulate autonomous cellular proliferation through interactions with cell surface receptors on secreting cells, a mechanism of autocrine stimulation of cell growth.

The possibility is important that regulation by growth factors might be important in transformation by viruses because common metabolic and morphologic features were shared in cells stimulated by each. The discovery of specific genes responsible for initiation and maintenance of transformation (oncogenes) associated with acute transforming retroviruses (v-oncs), and the additional discovery that the retroviral oncogenes were derived from normal cellular homologues (c-oncs, proto-oncogenes) of the oncogenes, focused additional attention on mechanisms of regulation of normal and neoplastic growth and suggested that cellular oncogenes encode protein products essential for the regulation and stimulation of pathways of normal cell growth and differentiation. Proto-oncogenes now have been identified and mapped to different chromosomes.

In the past decade multiple polypeptide growth factors have been isolated and sequenced; these include epidermal growth factor, insulin-like growth factors, interleukin-2, transforming growth factors, nerve growth factor, and the platelet-derived growth factor, as well as others less well defined. It is clear that major progress has been made in establishing biochemical roles for growth factors, growth factor receptors, and transforming proteins in normal and abnormal cell growth. Available information also suggests normal functions for PDGF in inflammation and repair.

Dr. Patricia A. D'Amore discussed "Sources and Actions of Growth Factors." Growth- and differentiation-inducing activities have been identified from a wide variety of tissues. These biologic activities have been characterized and purified to varying degrees. Only those growth factors that have been purified to homogeneity were discussed in this presentation. There are at least six major classes of biologically active growth factors. (1) Nerve growth factor (NGF), a polypeptide of variable molecular weight $(30,000$; $116,000 ; 130,000)$, has been isolated from a large number of tissues with mouse submaxillary gland as a major source. In vitro it supports the growth and differentiation of sympathetic nerve cells; in vivo it has been shown to influence sympathetic, sensory, chromaffin, and mast cells. (2) Epidermal growth factor (EGF) is a 6100 dalton polypeptide growth factor isolated from mouse submaxillary gland. EGF stimulates the proliferation of fibroblasts and epithelial cells in culture and has been shown to cause precocious eyelid opening and incisor eruption in newborn animals. (3) PDGF is a glycosylated polypeptide of $28-32,000$ daltons. PDGF was initially isolated from platelets and recently has been reported to be produced by a number of normal and transformed cell types. PDGF stimulates the proliferation of fibroblasts and fibroblasts in vitro and has been postulated to be involved in the response to injury in large blood vessels.

(4) Insulin-like growth factors (IGF) are a class of polypeptides and include somatomedin-C (also known as IGF-1) and multiplication stimulation activity (MSA; also known as IFG-II). The IGF's have been demonstrated in a number of tissues, although liver is thought to be the site of synthesis. The somatomedins have been shown to stimulate DNA synthesis in most cultured cell types examined. In vivo they are thought to play a role in normal growth since their production is stimulated by growth hormone. (5) The interleukins are a class of related polypeptides purified from blood leukocytes. The best characterized of these molecules are interleukins 1 and 2. Interleukin-1 (IL-2) has a molecular weight of about 18,000 . It has been shown to mediate a variety of effects including the bodyaches associated with infections and stimulation of connective tissue cells. Interleukin2 (IL-2) is a polypeptide factor released by leukocytes. Isolated from human cells it has a molecular weight of 15,000; isolated from murine cells the molecular weight is 30,000 . IL-2, also known as T-cell growth factor, supports the in vitro growth of Tcells.

(6) Heparin-binding growth factors (HBGF) are the most recently described group of biologically active polypeptide growth factors. Purified on the basis of the ability to stimulate Balb/c 3T3 cell DNA synthesis and the proliferation of vascular endothelial cells, these factors all share the property of a strong affinity for heparin. The group of factors encompases two subclasses that are characterized by differences in their isoelectric points, the degree of their affinity for heparin, and their amino acid composition. The class 1 heparin-binding factors are anionic (pI's of 5.0) and elute from immobilized heparin with about $1 \mathrm{M} \mathrm{NaCl}$. They have been isolated from a variety of neural tissues including retina, brain, and hypothalamus. The class 2 heparin-binding factors are cationic (pl's of 8-10) and they elute from immobilized heparin with about $1.5 \mathrm{M} \mathrm{NaCl}$. These factors have been isolated from tumors (hepatoma and chondrosarcoma), cartilage, macrophages, placenta, as well as from neural tissues. Specific differences between the amino acid compositions of the two classes of factors suggest that the two classes, although related, may be distinct. The target cell specificity of these factors has not been studied extensively. A number of reports indicate that at least some of these factors possess the ability to stimulate the formation of new blood vessels in vivo.

The "Factors Affecting Lung Metabolic Function" were discussed by Dr. C. Norman Gillis. Pulmonary endothelial cells take up and metabolize many important circulating vasoactive and other hormones. These processes have been widely studied, and it is now clear that many reflect transport sites (receptors?) and enzymes on the endothelial luminal membrane. For this reason and because endothelial cells also are sites of morphologic and functional change early in response to lung injury, the proposal has been made that measurement of these metabolic processes of uptake and metabolism, i.e. removal functions, also may be sensitive indices of the damage caused by such injury. In experiments to evaluate this proposal, many groups now have shown that removal of a variety of vasoactive substrates, including serotonin, substrates for angiotensin converting enzyme (ACE), and prostaglandins, is decreased by a variety of experimentally induced lung injury models. These include radiation, normobaric hyperoxia, endotoxin or monocrotaline, or bleomycin toxicity. Interestingly, in many of these cases lung metabolic functions were depressed before there was morphologic evidence of damage. Accordingly, it is now accepted that the injured lung has decreased metabolic function and, furthermore, that such depressed function may predict impending clinically evident injury. Less clear, however, is the precise cause of such decreased removal capacity. It could reflect diminished convection or delivery of substrate to the site of uptake, a diminished endothelial surface for uptake, or frank dysfunction of the endothelial transporter metabolic sites.

In order to develop methods that will permit separation of the influence of these factors, Gillis made extensive use of the mathematical model of saturable removal function proposed by Linehan, Dawson, and their colleagues in Wisconsin. This model utilizes information from indicator dilution outflow curves and allows calculation of apparent kinetic parameters (i.e., $V \max _{\mathrm{app}}$ and $\mathrm{Km}_{\mathrm{app}}$ ) for substrates (e.g. serotonin), and also the influence of heterogeneity of transit times or path lengths in the determination of overall removal. Since removal is proposed as an extensive function of endothelium, $\mathrm{Vmax}_{\mathrm{app}}$ should indirectly reflect surface area; however, $\mathrm{Km}_{\mathrm{app}}$ is an intensive function and may therefore reflect the intrinsic endothelial metabolic function which is believed to be damaged by injury. With this technique the Wisconsin group has shown that the calculated $\mathrm{Vmax}_{\mathrm{app}}$ for 5-HT uptake is uninfluenced by altered flow, perfusion hetero- 
geneity, or the $\mathrm{pH}$ range $7.2-8.0$ but is depressed by embolization, which presumably diminishes the perfused surface area. Gillis found that the $\mathrm{Km}_{\mathrm{app}}$ and $\mathrm{Vmax}_{\mathrm{app}}$ for benzoyl-phe-ala-pro (BPAP, a synthetic substrate for angiotensin converting enzyme in isolated perfused rabbit lungs, were unchanged by altered flow in lung preparations in which zone II conditions $\left(\mathrm{P}_{\mathrm{pa}}-\mathrm{P}_{\mathrm{alv}}=\right.$ constant) were maintained. On the other hand, high lung volumes produced by raising alveolar pressure while perfusion pressure was held constant decreased Vmax $\mathrm{mapp}_{\text {, }}$ presumably by compressing the distensible vessels of the alveolar surface, thus reducing perfused surface area. In this case the BPAP V $\max _{a p p}$ was depressed significantly while $\mathrm{Km}_{\mathrm{app}}$ was uninfluenced.

These data lead to the conclusion that the Vmax for at least one removal function, namely the hydrolysis of BPAP which reflects the activity of luminal endothelial converting enzyme, is unchanged by many experimental variables and, in this sense, is likely to be of value in separating convective from kinetic influences on removal. In order to undertake a direct correlation between the $\operatorname{Vmax}_{a p p}$ for BPAP and capillary surface area, Gillis' colleagues at Yale, Drs. Pitt and Lister in collaboration with Drs. Paul Davies and Lynn Reid at Harvard, undertook a study in which they followed several parameters of lung function in a series of fourteen sheep from birth to 6 months of age. In each animal several determinations of $\mathrm{Vmax}_{\mathrm{app}}$ for BPAP and DL were made. In addition, at various ages during the 6 months lungs were removed and stereologic determinations of capillary volume and surface area carried out. These studies indicated that there is a close correlation between morphometrically determined surface area and either the $\mathrm{DL}_{c o}$ or the $\mathrm{Vmax}_{\mathrm{app}}$ for BPAP. It was emphasized that the log-log plots of $V \max _{a p p}$ for BPAP lung volume had an alometric constant of more than 1.7, thus correlating with the relatively greater growth of surface area to capillary volume in the developing animals. Gillis believes it is possible to utilize this combination of techniques to study not only the physiology but also the pathophysiology of the lung microcirculation. Current work is directed toward the use of these techniques in acute, diffuse lung injury.

"Pulmonary Vascular Inflammatory Reactions" were discussed by Dr. Peter M. Henson. Most diseases of the lung of major importance have, as a significant component of their pathogenesis, an element of the inflammatory process. These include pulmomary vascular diseases as well as asthma, emphysema, pneumonia, interstitial lung diseases, hypersensitivity pneumonitides, and respiratory distress syndromes. If inflammation is defined as "a nonspecific response of tissues to injury," it is not difficult to see why this would be so. Normally, inflammation is beneficial and protective, ultimately leading to repair, regeneration, and return to normal structure and function. By contrast, in the context of clinical disease it can be injurious in itself and also can result not in resolution but fibrosis. For these broad reasons it would seem important to gain a detailed understanding of the inflammatory process in the lung and particularly of its normal mechanism of resolution in order to better contivl its progress in patients.

A simple concept of inflammation would include: (1) alteration in blood vessels, (2) emigration of inflammatory cells, (3) secretion of constituents from inflammatory cells, (4) removal of inflammatory cells and debris, (5) replication of parenchymal cells and repair of the tissue.

To exemplify the issues raised above, Henson briefly discussed the processes of emigration of inflammatory cells, in particular the neutrophil, into the lungs. Emphasis was placed on areas which need further investigation. Neutrophils must first interact with the vascular endothelium. Chemotactic factors produced extravascularly in the airspaces or interstitium are presumed to gain access to the vessel, although the mechanisms for this are unknown. Here they stimulate the passing leukocytes to develop adhesive properties which lead them to bind to the endothelial surface. Other influences, interleukin 1, for example, also may act on the endothelium so that it directly, or indirectly, also is adhesive. Once adherent ther neutrophils must then find the endothelial cell junctions and penetrate these. The mechanisms are not known.

The next barrier faced by the emigrating cell is the basement membrane. Data from artificial connective tissue barriers in vitro suggest strongly that the migratory cells digest their way through such barriers by expression of proteolytic activity as a consequence of chemotactic factor stimulation. After traversing a second basement membrane, the neutrophil encounters the alveolar or airway epithelium. Again, using artificial epithelial barriers, it has been shown that neutrophils can penetrate such barriers without changing the electrical resistance across such monolayers (i.e. without increasing the permeability to ions). On the other hand, if the neutrophil remains in contact with the epithelium, it does induce an increased permeability, but one which is reversible. These data suggest that in vivo neutrophil emigration does not necessarily lead to enhanced endothelial or epithelial permeability and is consistent with observations in sheep or rabbits of leukocyte emigration without demonstrable permeability changes. The circumstances that do lead to alteration of vascular integrity, therefore, gain prominence as areas for investigation.

Henson has been particularly interested in the effects of bacterial endotoxin, [lipopolysaccharide (LPS)], in this regard. LPS enhances neutrophil adhesion to endothelial cells in vitro and to pulmonary microvascular endothelium in vivo by virtue of a direct effect on the leukocyte. In addition, LPS "primes" neutrophils so that they are more responsive to other stimuli with regard to production of oxygen radicals and secretion of proteases. The combination of LPS and chemotactic stimuli would, therefore, be expected to enhance and prolong neutrophil interaction with lung vessels and to promote greater release of toxic products. This combination, in fact, induces increased accumulation of albumin in the lungs of experimental animals and directly causes neutrophil-dependent toxicity to endothelial cells in culture. It was suggested that combinations of chemoattractant and other neutrophil stimuli and enhancing facing (such as LPS) may be important contributors to endothelial injury in vivo.

Certainly the basic mechanisms of the normal inflammatory response in the lung are important areas of investigation. The processes are involved in many forms of lung disease and are responsive to a number of forms of therapeutic intervention. As additional understanding is gained of these mechanisms, it is hoped that rational treatments will allow preservation of the essential, beneficial aspects of inflammation, while at the same time reducing the injurious manifestations and minimizing the clinical disease.

The "Interaction of Endothelial Cells with Platelets and Plasma Clotting Factors" was discussed by Dr. Douglas B. Cines. Following vascular trauma bleeding stops when a platelet-fibrin clot forms at the injured site. In healthy individuals clot formation is restricted to the affected area. There is extensive experimental evidence indicating that this localization results from exposure of circulating blood elements to structures normally confined within subendothelial portions of the vessel wall. These vascular components are required to initiate platelet adherence to the site of trauma and to activate plasma clotting proteins which generally circulate as inactive molecules. This hypothesis is supported by the clinical association of excessive bleeding with deficiencies or dysfunction of clotting factors (such as von Willebrand's factor) or cell surface receptors (such as platelet glycoprotein Ib) involved in the process of platelet adherence to these subendothelial structures. Endothelial cells may participate in this process by synthesizing von Willebrand's factor, as well as several structural components of the basement membrance matrix including collagen, laminin, fibronectin, and several types of sulfated mucopolysaccharides that may be involved in platelet aggregation.

It has been assumed that exposure of subendothelial cell structures also is involved in the far more common thrombotic 
disorders that develop in man in the absence of overt trauma. According to this view endothelial cells do not participate directly in the pathogenesis of thrombosis. Rather endothelial cells play a critical role in preventing the formation of intravascular clots under physiologic conditions by several mechanisms. First, endothelial cells secrete prostacyclin that inhibits certain steps in the activation of platelets. Second, endothelial cells secrete heparan sulfate that accelerates the capacity of antithrombin III to inactivate thrombin. Third, endothelial cells elaborate thrombomodulin, a protein that binds thrombin and changes its preferred substrate from coagulation factors V, VIII, and fibrinogen to protein $\mathrm{C}$. In turn activated protein $\mathrm{C}$ inhibits the function of activated factors V and VIII. Finally, endothelial cells synthesize both urokinase-type and tissue-type plasminogen activators that accelerate the dissolution of fibrin clots. Therefore, clot formation is unlikely to occur when a continuous monolayer of endothelial cells line the blood vessels and fully express these antithrombotic functions.

Theoretically any process that injures endothelial cells would disrupt these anticoagulant functions that predominate under physiologic conditions. However, to date, no thrombotic disorders have been identified that unequivocally result from an inherited or acquired dysfunction of endothelial cells alone. Indeed, until recently, there has been little interest in the possibility that endothelial cells could be directly involved in the initiation of the thrombotic process under any circumstances.

The cause of thrombosis in most clinical conditions remains unknown. Neither evidence of vascular trauma nor abnormalities of platelets or coagulation proteins can be identified in most patients who develop thromboses. Recently some experimental evidence has been obtained that indicates that alteration of the physiologic function of endothelial cells may exert a direct procoagulant effect. For example, platelets derived from normal individuals will adhere to endothelial cells that have been preexposed to thrombin in vitro or to endothelial cells that have been infected with SV-40 virus. Platelets also will adhere to cultured endothelial cells that have been incubated with antiendothelial cell antibodies or immune complexes present in the sera of patients with active systemic lupus, a disorder associated with vasculitis and thrombosis.

In addition, cultured endothelial cells are capable of synthesizing tissue factor, a lipid-protein complex responsible for the initiation of the extrinsic pathway of coagulation. Tissue factor is produced by the endothelium following exposure to thrombin, endotoxin, endothelial cell antibodies, or immune complexes. Moreover, endothelial cells in culture express receptors on the cell surface for coagulation factors, XI, IX, and IXa. Specific binding sites for fibrinogen and certain fibrin degradation products have also been identified on endothelial cells.

Finally, endothelial cells produce a specific inhibitor of urokinase and tissue-type plasminogen activator. Changes in the level of inhibitor at sites of trauma or disease may play a critical role in the regulation of fibrinolysis. Indeed, increased levels of inhibitor and/or decreased levels of plasminogen activator have been found in the sera of a high percentage of patients with idiopathic venous thromboses.

Therefore, recent experimental evidence points to the possibility that perturbations of endothelial cell functions that have been identified in vitro could initiate or accelerate thrombotic processes. However, it is not known if endothelial cells within the vessel walls themselves display similar surface receptors or synthesize similar procoagulant or anticoagulant factors as endothelial cells in culture. Moreover, the function of endothelial cells may be modified by other plasma components or products secreted by other cells within the vessel wall. Therefore, additional research will be required to determine whether inherited or acquired disorders of endothelial cell function play an important role in the pathogenesis of clinically significant bleeding or thrombotic disorders in man.

Session IV was entitled "Clinical Applications-Diagnosis and
Therapy" and was chaired by Dr. William F. Friedman. The "Detection of The Location of Changes in Pulmonary Vascular Tone" was discussed by Dr. John $\mathrm{H}$. Linehan. To provide insight into the longitudinal arrangement of pulmonary vascular resistance relative to vascular compliance, Linehan developed a venous occlusion technique in which the venous outflow from a lung lobe perfused with constant flow is rapidly occluded. The venous pressure initially rises rapidly followed by a slower and nearly linear increase in both arterial and venous pressures. To interpret these data, a compartmentalized hemodynamic model was employed which visualizes the vascular bed as three serial resistances separated by equal parallel compliances. In response to various vasomotor stimuli, it was found that serotonin, sympathetic nerve stimulation, hypoxia, and prostaglandin $F_{2}$ increased the arterial (upstream) pressure drop, while histamine, norepinephrine, epinephrine, and elevation of the cerebrospinal fluid pressure increased the venous (downstream) pressure drop. The pressure drop for the middle compartment, which may represent vessels located between the muscular arteries and vein, was not affected by the stimuli. Linehan concluded that the average pressure of the middle compartment is a microvascular pressure that can be used to evaluate the impact of vasoconstriction on the lobar microcirculation.

"Cardiac Catheterization, Quantitative Wedge Angiography, and Lung Biopsy" were the subjects discussed by Dr. Marlene Rabinovitch. The most fruitful assessment of the patient with pulmonary hypertension is one that is both diagnostic and prognostic as well as investigative. In this way the probability of predicting the outcome will be potentiated by the possibility of favorably altering the course of the disease. Rabinovitch has been using a three-pronged diagnostic approach using cardiac catheterization, quantitative wedge angiography, and lung biopsy. She pointed out both the merits and pitfalls of each technique and showed how the latter become particularly "weighty" when any one diagnostic approach is used in isolation.

Cardiac catheter studies have given us the security of a reference point, a "number," but the insecurity that many confounding variables affect its significance. Some of the variables are easily identified, e.g. hypoxia, state of sedation, hematocrit, bronchial blood flow; others, such as the influence of age and genetic predisposition, are more difficult to assess.

Differentiating between the patient with reactive versus fixed pulmonary hypertension may also be possible in the catheter laboratory, but more long-term follow-up studies are necessary to determine which pharmacologic agents, which age group, and which degree of response are most predictive of a favorable longterm outcome. Better measurement of "absolute" pulmonary blood flow, in the future, might improve overall assessment.

Quantitative pulmonary wedge angiography, using the balloon occlusion technique, has given us a new insight into the severity of the structural features that are less affected by the variables that confound the measurement of pulmonary vascular resistance. Three features of each wedge study always should be taken into consideration-the rate of taper, the background filling of peripheral vessels, and the pulmonary circulation time. Rate of taper can be falsely abrupt (i.e. falsely indicative of advanced vascular disease) in the presence of a pulmonary artery band or pulmonary stenosis if, in either case, there is poststenotic dilatation. It can be falsely gradual (i.e. falsely indicative of normal or minimal disease) when the artery is constricted or when the changes of intimal hyperplasia have affected the larger vessels. Background filling can be scant if the injection is technically poor and pulmonary circulation time can be prolonged when there is pulmonary venous stenosis. Application of techniques using densitometry show new promise.

Rabinovitch has found features evident on a lung biopsy specimen, even when analyzed by frozen section, to be generally predictive of reactivity and reversibility of pulmonary vascular disease. However, among candidates for a Fontan procedure, i.e. a right atrial to pulmonary artery anastomosis, $50 \%$ with poten- 
tially reversible pulmonary vascular changes (medial hypertrophy) will have increased pulmonary vascular resistance in the postoperative period; unfortunately, we do not know which $50 \%$. Also there are some individuals whose biopsies are misleading. Particularly worrisome are rare patients with only medial hypertrophy who behave postrepair of a simple congenital heart defect, e.g. a ventricular septal defect, as though they have fixed elevation in pulmonary vascular resistance. Ultrastructural studies have revealed a few clues; there are striking endothelial changes and/ or increased connective tissue proteins (elastin, collagen) in the subendothelium.

Rabinovitch is currently directing her research efforts, both clinically and in the laboratory, toward determining the functional significance of these abnormalities. In studies using scanning electron microscopy changes have been identified in the surface characteristics of pulmonary arteries which suggest abnormal interaction with blood elements. On transmission electron microscopy there is an increase in those cytoplasmic components of endothelial cells which are associated with protein synthesis. According to these findings many patients with pulmonary hypertension either associated with congenital heart defects or idiopathic have high circulating factor VIII levels. Further exploration is indicated of functional properties of endothelial cells which might reflect abnormal interaction with blood elements, e.g. assessment of platelet activating factor. Further, experimental models have been developed to study the evolution of elastin degradation and resynthesis in the pathogenesis of fixed pulmonary hypertension.

In endothelial and smooth muscle cell culture studies a method was developed of pulsating cells at high pressure, simulating the condition of a congenital heart defect with a left-to-right shunt. Studies of the structural properties of these cells reveal that they are similar to those observed in vivo. Therefore, this approach is aimed at determining abnormalities in endothelial-smooth muscle interaction, particularly with regard to stimulation of smooth muscle hypertrophy, hyperplasia, and synthesis of connective tissue proteins.

The "Echo Doppler Evaluations of Patients Suspected of Having Pulmonary Hypertension" was discussed by Dr. David J. Sahn. A variety of ultrasound methods have been developed in an attempt to achieve noninvasive estimation of pulmonary artery pressure as an indicator of pulmonary hypertension. This report reviewed the relative accuracy, reproducibility, and applicability of these methods.

The history and application of right-sided systolic time intervals and the difficulties of reproducing measurements between observers and determining the patient with inoperable pulmonary hypertension was discussed initially. With the advent of Doppler echocardiography, new methods became potentially available for evaluation of patients with pulmonary vascular disease. Separate calculation of QP:QS as indicators of pulmonic and systemic resistance have been attempted. These volume flow methods involve several assumptions, mainly about the area and profile of flow. They, however, certainly can be applied to differentiate large shunts from small shunts and to test patients' vasoreactivity to oxygen stimulation. Other Doppler methods have been used to estimate right heart or pulmonary artery pressure. One method, initially described in Japan, involves calculation of the acceleration time and ejection time of the Doppler recording of the pulmonary artery flow velocity waveform. While this methodology appears to provide time intervals that are easier to recognize than pulmonary valve closure by $\mathrm{M}$ mode, as a time interval method, especially for the short acceleration time, the approach appears to have difficulties in reproducibility of measurements between settings or observers.

Another method that appears quite promising for the evaluation of pulmonary arterty pressure in patients who do not have right ventricular outflow tract obstruction, is the estimation of right ventricular pressure from the velocity of tricuspid insufficiency as estimated by the Bernoulli relationship. This assumes a discrete orifice behavior for the tricuspid valve in tricuspid insufficiency. Tricuspid insufficiency is quite common as a silent associated lesion in many patients with congenital heart disease, and by estimating the maximal velocity of right ventricle to right atrial flow, the RV:RA gradient therefore can be estimated. This, when added to an estimate of central venous pressure, serves to estimate RV pressure.

Lastly, new applications of color Doppler flow mapping appear to be quite promising for imaging the direction of shunting through intracardiac defects and for evaluating the location and directional nature of shunting to aid the prediction and management of the patient at risk for pulmonary vascular disease. The sensitivity of these color flow methods for detecting right-to-left shunting can be improved by performing them in combination with contrast echo injection.

The use of ultrasound for assessment of pulmonary vascular bed still presents problems, but new methods and approaches continue to evolve in this rapidly developing field of medical imaging.

Dr. John T. Reeves presented a report on the "Treatment of Primary Pulmonary Hypertension." Primary (or unexplained) pulmonary hypertension is a rare but highly fatal disease which may afflict young adults, most often women. Its pathogenesis is unknown and its natural history is variable. However, in some patients the pulmonary vascular abnormality as described by pathologists is primarily medial hypertrophy of pulmonary arterioles, suggesting a reversible component of the disease. Also, in some patients marked reduction in pulmonary vascular resistance with the acute administration of vasodilator agents at cardiac catheterization indicates that a large part of the hypertension is due to vasoconstriction. Yet in others with the disease both the morphologic findings (intimal sclerosis, adventitial fibrosis, vessel loss) and the failure of acute vasodilation are compatible with the concept of a fixed, irreversible pulmonary hypertension. Reeves' hypothesis is that vasoconstriction precedes and contributes to the development of fixed obstruction. The hypothesis should be examined because, if true, it would imply that treatment be focused on those patients with a large vasoconstrictive component to improve them and to slow the progress of the disease.

The published reports (since 1978) were reviewed of 117 patients in whom individual cardiac catheterization data were reported before and after vasodilator challenge. Before vasodilator challenge the calculated pulmonary vascular resistance ranged from approximately 4 to 60 Wood units and the median values was 16.7 U. Following the administration of an acute vasodilator the change in pulmonary vascular resistance ranged from an increase of approximately $25 \%$, to a decrease of $70 \%$, with a median change of $-25 \% ; 53$ patients showed a decrease of $30 \%$ or more. With the most popular agents the median changes in resistance were: for the calcium antagonists $(n=46)$, $-30 \%$, for hydralazine $(n=23),-35 \%$, and for diazoxide $(n=$ $18),-35 \%$. Captopril $(n=17)$ gave a relatively poor response, $-7 \%$. Among the 117 reported patients, there were 37 in whom specific clinical and/or hemodynamic benefit was noted, lasting 3 months or more. For the purposes of discussion the assumption was made that the remaining 80 patients did poorly. However, 33 of the 37 patients had, with acute vasodilator challenge, a decrease in resistance of $30 \%$ or more. Repeat hemodynamics suggested that sustained reduction in resistance was achieved with 3 to 48 months of therapy. Five patients having multiple heart catheterizations over 1 to $4 \mathrm{yr}$ indicated that reductions in resistance for at least 3 months were likely to be sustained. In this disease long-term benefit occurs in a minority of patients and is never certain. However, there is a subset, characterized by a large reduction of pulmonary vascular resistance during acute vasodilation, in whom long-term vasodilator therapy is associated with months and even years of improvement. At present therapeutic efforts should be focused on this subset of patients.

The "Therapy of Secondary Pulmonary Hypertension" was 
discussed by Dr. Lewis J. Rubin. Pulmonary hypertension can complicate a variety of childhood diseases that primarily affect the structure or function of the lung. Therapy is directed primarily at ameliorating hypoxic and hypercarbic pulmonary vasoconstriction by improving intrapulmonary gas exchange using bronchodilators, antibiotics, corticosteroids, and low-flow oxygen. Diuretics have not been demonstrated to improve substantially hemodynamics or oxygen transport in cor pulmonale, and decreasing right heart filling pressures may be deleterious by producing a decrease in cardiac output. Cardiac glycosides appear to improve right ventricular function only when coexistent left ventricular dysfunction is present.

The rationale for vasodilator therapy for secondary pulmonary hypertension is based on the observations that an increased right ventricular afterload, produced by constriction of the pulmonary resistance vessels, is the cause of the progressive deterioration in right ventricular function in these patients and contributes to the impairment in oxygen delivery to the peripheral tissues. Several systemic vasodilators have been shown to reduce pulmonary vasomotor tone in experimental pulmonary hypertension. Clinical studies evaluating the effects of vasodilators in patients with secondary pulmonary hypertension have demonstrated reductions in pulmonary artery pressure and pulmonary vascular resistance at rest and during exercise, improvements in right ventricular ejection fraction measured by radionuclide angiocardiography, and increases in oxygen transport. However, no consistent pattern of response is noted, and systemic side effects preclude continuing therapy in some patients.

Additionally, some vasodilators, particularly nitrates and the calcium channel blockers, worsen $\mathrm{V}_{\mathrm{A}} / \mathrm{Q}$ relationships and may aggravate hypoxemia. Finally, the clinical significance of the hemodynamic effects of vasodilator therapy, in terms of improved activity tolerance or survival, have not been addressed. Nevertheless, this form of therapy may hold promise as an adjunct to conventional therapy, particularly if more selective pulmonary vasodilator agents can be identified.

The last presentation by Dr. Robert Wolfe concerned "Pulmonary Hypertension at Altitude." The pulmonary vascular effects of acute and chronic exposure to high altitude in North American infants and children have not been well characterized. Consequently, the Pediatric Cardiology Division of the University of Colorado School of Medicine has utilized acute hypoxic challenge in conjunction with diagnostic cardiac catheterization for more than $10 \mathrm{yr}$. The patients have resided chronically at altitude from 3,000-11,000 feet and were studied for a wide variety of indications.

The acute $(10-\mathrm{min})$ response to $16 \%$ inspired oxygen in patients with normal pulmonary vascular resistance (PVR) in Denver, and little or no evidence of pulmonary hypertension under more hypoxic conditions, was minimal $(0-20 \%$ increase in PVR). Patients with clinical or historical evidence of hypoxic pulmonary hypertension (50-500\% increase in PVR) were labeled as hyperreactive. Two pediatric pulmonary hypertensive conditions have been studied, as have a wide variety of children with congenital heart disease.

Symptomatic high altitude pulmonary hypertension of infancy is a potentially lethal disease state. It has been characterized in infants chronically residing at or above 10,000 feet.

Seven infants with structurally normal hearts presented with symptoms of congestive heart failure responding to oxygen and anticongestive therapy. When asymptomatic they were studied in Denver and found to have normal to minimally increased pulmonary artery pressure, but all had dramatic increases in pulmonary arterial pressure in response to acute hypoxia (400$500 \%$ ) with resultant suprasystemic pulmonary hypertension. These infants die unless they move to a lower altitude. All have shown regression of right ventricular hypertrophy after moving to a lower altitude.

High altitude pulmonary edema was a second entity discussed by Wolfe. Seven children with reentry high altitude pulmonary edema were similarly studied and found to have somewhat less dramatic acute hypoxic responses (200-300\%) and rarely had suprasystemic pulmonary artery pressures. No known deaths have occurred in this group but recurrent reentry high altitude pulmonary edema has significantly affected their lifestyles.

Whether or not children with unoperated congenital heart disease have higher pulmonary vascular resistance at altitude is controversial and difficult to study in a controlled manner. However, a strong tendency appears to exist for altitude-related elevation in pulmonary vascular resistance, especially in patients with left to right shunts. However, after total surgical correction, the majority return to normal values of pulmonary vascular resistance unless they have one or more of the following risk factors: Down syndrome, chronic residence above 10,000 feet, chronic lung disease, upper airway obstruction, abnormal thoracic function, hypoventilation, or sleep apnea. Thus the combination of the natural mild hypoxia of chronic altitude residence plus associated hypoxic disease states appear to increase pulmonary vascular reactivity.

\section{EPILOGUE}

Dr. Fishman contributed final thoughts concerning the workshop and a summary of its contents. He concluded that for the audience of clinical investigators in pediatrics growth and development of the pulmonary circulation is a natural area of inquiry. Although it is true that important anatomical beginnings have been made in understanding restructuring of the pulmonary circulation, these have only whetted the scientific appetites for greater insights into mechanisms. The control of the ductus arteriosus continues to be a fertile field, especially when account is taken of the divergent responses of the ductus and the pulmonary arterial segments immediately adjacent when exposed to hypoxia or hyperoxia. Larger questions such as the integrated responses of the pulmonary circulation to the onset of breathing and the respective roles of neurohumoral versus mechanical influences also have not quite fallen into place.

The transfer from the normal to the abnormal pulmonary circulation provides a cornucopia of questions and opportunities. The role of the expanded collateral circulation to the lungs and the mechanisms controlling proliferation of this systemic circulation are largely enigmatic. Although pulmonary vasodilators are receiving extensive trials both in this country and abroad, it is remarkable how little is understood about determinants of pulmonary vasomotor tone and the mechanisms involved in the pharmacologic induction of an active change in pulmonary vascular resistance.

It is clear that we are living in an age of cellular and molecular biology on the one hand and of integrative biology and neuroscience on the other. The concepts and techniques of these lines of research are destined to have large impacts on research into the regulation of the pulmonary circulation. Local mediators, growth factors, cell-cell interactions, and plasma-endothelial interplay were all discussed during the workshop described herein. The challenge now is for those familiar with current concepts and techniques with respect to the pulmonary circulation to extend their horizons and incorporate the lessons from the advancing frontiers of science into their studies of the pulmnary circulation, its normal behavior, and the modifications induced by disease.

\section{PARTICIPANTS}

Robyn J. Barst, M.D.

Assistant Professor of Pediatrics and Pharmacology

New York Medical College

Valhalla, NY 
William Berman, Jr., M.D.

Professor of Pediatrics

Unviersity of New Mexico

Alburquerque, NM

Kenneth L. Brigham, M.D.

Joe and Morris Werthan Professor of Investigative Medicine

Director, Pulmonary Circulation Ctr.

Vanderbilt University School of Medicine

Nashville, TN

Peter H. Burri, M.D.

Professor of Anatomy

Anatomisches Institut

Universitat Bern

Bern, Switzerland

Douglas B. Cines, M.D.

Assistant Professor of Medicine

Department of Medicine

Hematology-Oncology Section

Hospital of the University of Pennsylvania

Philadelphia, PA

Patricia A. D'Amore, Ph.D.

Assistant Professor of Pathology

Department of Surgical Pathology

Children's Hospital Medical Center

Boston, MA

Michael Heymann, M.D.

Professor of Pediatrics and Obstetrics and Reproductive Science

Department of Pediatrics

Cardiovascular Research Institute

University of California

San Francisco, CA

Julien I.E. Hoffman, M.D.

Professor of Pediatrics and Physiology

Cardiovascular Research Institute

University of California

San Francisco, CA

Thomas F. Deuel, M.D.

Professor of Medicine and Associate Professor of Biological Chemistry

Hematology Division

Washington University

Medical School

Jewish Hospital

St. Louis, MO

Alfred P. Fishman, M.D.

William Maul Measey Professor of Medicine

Director, Cardiovascular Pulmonary Division

Hospital of the University of Pennsylvania

Philadelphia, PA

William F. Friedman, M.D.

J. H. Nicholson Professor and Executive Chairman

Department of Pediatrics

U.C.L.A. School of Medicine

Los Angeles, CA

C. Norman Gillis, Ph.D.

Professor of Anesthesiology and Pharmacology

Department of Anesthesiology

Yale University

School of Medicine

New Haven, CT
Peter M. Henson, Ph.D., D.V.M.

Professor of Pathology and Medicine

Department of Pediatrics

National Jewish Hospital and Research Center

Denver, $\mathrm{CO}$

John T. Reeves, M.D.

Professor of Medicine

Department of Medicine

University of Colorado Medical Center

Denver, $\mathrm{CO}$

Lynne M. Reid, M.D.

S. Burt Wolbch Professor of Pathology

Harvard Medical School

Chairman, Department of Pathology

Children's Hospital Medical Center

Boston, MA

J. Michael Kay, M.D.

Professor of Pathology

Department of Pathology

McMaster University

Hamilton, Ontario, Canada

Ivan F. McMurtry, Ph.D.

Associate Professor of Medicine

Department of Medicine

University of Colorado Health Sciences Center

Denver, CO

Marlene Rabinovitch, M.D.

Associate Professor of Pediatrics and Pathology

University of Toronto

Department of Cardiology

Hospital for Sick Children

Toronto, Ontario, Canada

Abraham M. Rudolph, M.D.

Neider Professor of Pediatric Cardiology

University of California

Cardiovascular Research Institute

San Francisco, CA

Una S. Ryan, Ph.D.

Research Professor of Medicine

Department of Medicine (D-58)

Univ. of Miami, School of Medicine

Miami, FL

David J. Sahn, M.D.

Professor of Pediatrics

University of California, San Diego Medical Center

San Diego, CA

Eveline E. Schneeberger, M.D.

Associate Professor of Pathology

Harvard Medical School

Department of Pathology

Massachusetts General Hospital

Boston, MA

Claude Lenfant, M.D.

Director

National Heart, Lung and Blood Institute

Bethesda, Maryland 
John H. Linehan, Ph.D.

Professor of Mechanical and Biomechanical Engineering Department of Biomedical Engrg.

Marquette University

Milwaukee, WI

Lewis J. Rubin, M.D.

Head, Pulmonary Division

Associate Professor of Medicine

University of Maryland School of Medicine

Baltimore, MD

Norman Talner, M.D.

Professor of Pediatrics

Chief of Pediatric Cardiology

Yale University School of Medicine

New Haven, CT

C. Richard Taylor, Ph.D.

Alexander Agassiz Professor of Zoology

Concord Field Station

Harvard University

Bedford, MA

Prof. dr. C. A. Wagenvoort

Professor of Pathology
Department of Pathology

Academic Medical Center

Amsterdam, The Netherlands

Robert R. Wolfe, M.D.

Associate Professor of Pediatrics

University of Colorado Medical Center

Denver, $\mathrm{CO}$

\section{NATIONAL HEART LUNG BLOOD INSTITUTE} STAFF

Suzanne Hurd, Ph.D.

Director, Division of Lung Diseases

Zena McCallum

Division of Heart and Vascular Diseases

Barbara Packard, M.D., Ph.D.

Director, Division of Heart and Vascular Diseases

Carol Vreim, Ph.D.

Chief, Interstitial Lung Diseases Branch 